\title{
Il Perdono Come Problema Filosofico
}

\section{Maria Grazia Carnevale ${ }^{1}$}

\section{Riassunto}

In sintesi, il perdono può essere definito come un atto di memoria in grado di interrompere e, nello stesso momento, di far ripartire ogni volta in modo diverso la corrente ordinata della temporalità storica: occorre che il richiamo al passato sia presente nella ferita con la stessa forza che aveva nell'istante in cui essa è stata inferta. II perdono, sebbene costituisca "un potere riservato a tutti $e$ posseduto da tutti”, è e deve rimanere eccezionale e straordinario: non potrà mai essere né normale, né normativo, nonostante i numerosi tentativi del diritto di incorporarlo e, soprattutto, non dovrà nascondere la sua estrema fragilità. Visto in questo contesto, il presente lavoro è dedicato al perdono come problema filosofico e giuridico.

Parole chiave: Perdono. Memoria. Temporalità. Diritto.

\section{O PERDÃO COMO PROBLEMA FILOSÓFICO}

\section{Resumo}

0 perdão pode ser definido como um ato de memória capaz de interromper e, ao mesmo tempo, de reiniciar cada vez de modo diverso a ordenada corrente da temporalidade histórica. Ocorre que a referência ao passado está presente na ofensa com a mesma força que tinha no instante em que foi cometida. 0 perdão, mesmo que seja "um poder reservado a todos e de propriedade de todos", é e deve permanecer excepcional e extraordinário: não pode jamais ser normal, nem normativo, apesar das inúmeras tentativas do Direito de incorporá-lo e, especialmente, não deve esconder a sua extrema fragilidade. Visto esse cenário, o presente texto é dedicado ao perdão como problema filosófico e jurídico.

Palavras-chave: Perdão. Temporalidade. Memória. Direito.

${ }^{1}$ Dottoranda in Diritto Europeo su base storico-comparatistica Facoltà di Giurisprudenza Università degli Studi di Roma Tre (Itália). mariagrazia.carnevale@virgilio.it 


\title{
FORGIVENESS AS A PHILOSOPHICAL PROBLEM
}

\begin{abstract}
Forgiveness may be defined as a memory act capable to interrupt and, at the same time, to steadily reopen the orderly flow of historic temporalness in a new way. But reference to the past is present in the offense with the same force it had had while being committed. Forgiveness, "although being a power reserved for all and of everyone's proprietary", is and shall keep exceptional and extraordinary: it can never be usual or normative despite the constant attempts of law to incorporate it, and it must especially not hide its extreme weakness. Under this setting this paper is dedicated to forgiveness as a philosophical and juridical problem.
\end{abstract}

Keywords: Forgiveness. Temporalness. Memory. Law. 
Il termine perdonare deriva dal latino ed è composto dal prefisso per, con efficacia rafforzativa, a sottolineare il carattere di offerta totale e incondizionata dell'azione stessa, e dal verbo donare, che rimanda al dono appunto. Esso designa un gesto umanitario con cui, vincendo i rancori e i risentimenti personali, si rimette una colpa, si rinuncia a ogni forma di rivalsa, di punizione o di vendetta nei confronti dell'offensore. Per estensione il perdono rimanda al significato di indulgenza verso le debolezze o le difficoltà altrui: quindi si riferisce a un sentimento di commiserazione e di benevolenza nei riguardi della persona che ha cagionato un'offesa. In senso più attenuato e con ben diversa valenza pratica viene usato per esprimere una scusa, intesa come venia, o anche come licenza, che si è soliti chiedere ad una persona della quale si teme di offendere col proprio linguaggio o comportamento, sia pure in lieve misura (e talora senza volerlo) la suscettibilità (per lo più in espressioni convenzionali di cortesia come "chiedo, domando perdono"). Da tali molteplici significati, uniti alla derivazione etimologica, inizia la nostra riflessione sul tema del perdono, volta dapprima a indagarne la vera essenza, per poi interrogarsi sulla possibilità e perfino sulla necessità di una regolamentazione giuridica del fenomeno.

Come sottolinea Olivier Abel, che si è a lungo interrogato su questo tema, collocare il perdono nella corretta cornice epistemologica significa solo offrire "una geografia dei dilemmi": tali interrogativi si innestano sul confronto di due atti di discorso, quello del colpevole ("Ti chiedo perdono") che enuncia la colpa commessa, a prezzo di un terribile lavoro di formulazione del torto, di una penosa ricostruzione dell'offesa e quello della vittima ("Ti perdono"), che viene supposta capace di pronunciare la parola liberatoria del perdono. Essi saranno sempre prigionieri di una

${ }^{2}$ Abel, O. Le perdon. Briser la dette et l'oubli. Paris: Autrement, 1992. p. 208-236. 
qualche forma di relazione (ad esempio tra la domanda e l'offerta di perdono, tra l'altezza del perdonare e la profondità della colpa...) in grado di produrre, però, evidenti dissimmetrie tra i soggetti coinvolti.

Il primo dato su cui soffermarsi è la pretesa affinità tra il dono e il perdono testimoniata dall'etimologia e dalla semantica di numerose lingue: in francese don-pardon, in spagnolo don-perdón, in portoghese dom-perdão, in inglese gift-forgive, in tedesco geben-vergeben... Dunque non c'è dono senza perdono, e non c'è perdono senza dono.

È utile anche riflettere sul ruolo rivestito dal munus (dono) nella società romana: esso rappresentava un onere, posto a carico di singoli, che solitamente ricoprivano cariche pubbliche (honores), per fini di pubblico interesse ${ }^{3}$. Il termine in questione che designa un facere, un'attività materiale, serve anche ad indicare per metonimia i beni prestati: esso implica la partecipazione del civis alla vita politica e sociale attraverso la gratuità del dono, che diviene parte di un obbligo giuridico (officium) per chi svolge funzioni pubbliche. Fin da subito è caratterizzato dall'onerosità e dall'obbligatorietà giuridica, quasi a sottolinearne la natura di contributo all'ordinato svolgimento della convivenza civile: siamo di fronte a un dono che perde la sua spontaneità per trasformarsi in dovere. La communitas (cum-munus $)^{4}$ che ne nasce è allora il luogo dove si scambiano i doni e si stabiliscono relazioni.

In realtà proprio l'elemento della gratuità, anziché accomunare dono e perdono, li differenzia all'interno del circuito dello scambio. Infatti a prima vista l'atto del donare sembra essere unilaterale e fondarsi totalmente sull'assenza di reciprocità tra donante e donatario: si lascia a qualcuno con intenzione liberale e senza riceverne niente in cambio un bene

${ }^{3}$ Definizione tratta dal Dizionario Giuridico Romano, a cura di F. del Giudice, edizioni Giuridiche Simone, 2010.

${ }^{4}$ Esposito, R. Communitas. Origine e destino della comunità. Torino: Einaudi, 1998. 
che si possiede o di cui si è proprietari. Un'analisi più attenta, tuttavia, spinge il dono verso il ristabilimento dell'equivalenza e ne mostra il carattere ambivalente ${ }^{5}$ : a questo proposito il testo classico di Marcel Mauss sul dono ${ }^{6}$ ci deve mettere in allerta. Mauss non contrappone il dono alla scambio, bensì alla forma commerciale dello scambio, al calcolo, allinteresse e, cosi facendo, valorizza l'eccesso munifico del dono che suscita il controdono e che interrompe l'ordine scontato degli affari, in cui l'aspettativa della reciprocità assume la forma dell'equivalenza monetaria. La controparte del dono non è ricevere, bensì dare in cambio, rendere, come testimonia un proverbio scandinavo citato dall'autore stesso: "ricambiare regalo con regalo". Quello che il sociologo esplora è un tratto "profondo ma non isolato; il carattere volontario, per cosi dire apparentemente libero e gratuito, e tuttavia obbligato e interessato di queste prestazioni"s: c'è una forza misteriosa contenuta nella cosa donata che fa sì che il donatario la ricambi e che le tre obbligazioni di dare, di ricevere, di rendere siano indissolubilmente legate tra di loro (ovvero "nelle cose scambiate nel potlàc c'è una virtù, che costringe $i$ doni a circolare, a essere dati e a essere ricambiati $\left.{ }^{\text {’x}}\right)$.

Invece, il gesto del perdono affinché sia autentico deve essere un atto assolutamente gratuito, senza corrispettivo o obbligo di ricambiare presente o futuro: il requisito della gratuità deriva dal disinteresse presente sia nella richiesta di colui che vorrebbe essere perdonato, sia nella manifestazione di volontà del concedente il perdono. Su questo punto si gioca la questione del vero dono, suscettibile di essere frainteso e tradito quando una qualsivoglia dinamica di calcolo gli si frappone: il donante

${ }^{5}$ Come sottolineato da E. Resta. Le regole della fiducia. Milano: Feltrinelli, 2009. p. 43.

${ }^{6}$ Mauss, M. "Saggio sul dono. Forma e motivo dello scambio nelle società arcaiche”. In: Teoria generale della magia. Torino: Einaudi, 1965. p. 135-292.

${ }^{7}$ MAUSS, M. op. cit., p. 156.

${ }^{8}$ MAUSS, M. op. cit., p. 157.

${ }^{9}$ MAUSS, M. op. cit., p. 227. 
che diviene interessato e che si aspetta una contropartita muta sé stesso in contraente, inserendo il suo relazionarsi con il donatario nella logica commerciale retributiva del pari valore. Del resto ci ricorda Seneca nel suo De beneficiis: "Non può chiamarsi beneficio quel che viene dato per lucro. "Darò tanto e riceverò tant'altro": questo è un mercato!10".

Infatti "ogni volta che il perdono è al servizio di una finalità, per quanto nobile e spirituale possa essere (riscatto o redenzione, riconciliazione, salvezza), ogni volta che esso tende a ristabilire una normalità (sociale, nazionale, politica, psicologica) attraverso un lavoro del lutto, attraverso una qualche terapia o ecologia della memoria, allora il perdono non è puro e neppure il suo concetto" 11 come afferma Jacques Derrida di fronte " $a$ tutte le scene di pentimento, di confessione, di perdono o di scuse che si moltiplicano sulla scena geopolitica a partire dall'ultima guerra e, in maniera accelerata, a partire da qualche anno"12. D'altronde egli sostiene che "il simulacro, il rituale automatico, l'ipocrisia, il calcolo o la scimmiottatura si sono inseriti spesso nella partita e si invitano da parassiti a quella cerimonia della colpevolezza"13 e ci avverte del pericolo che tale universale esigenza di memoria si pieghi alle istanze politiche dello Stato nazione, mentre dovrebbe rimanere una necessità morale della storia. Tuttavia il dono stabilisce un legame tra chi lo dà e chi lo riceve : "il dono diventa più prezioso, pur essendo sempre lo stesso, se inaugura una catena di relazioni" ${ }^{4}$.

Il superamento dell'idea stessa di perdono è del resto rappresentato dall'amore per i propri nemici : "Se amate quelli che vi amano che merito ne avrete? Anche i peccatori fanno lo stesso...Amate invece $i$ vostri nemici,

\footnotetext{
${ }^{10}$ Seneca, L. A. De beneficiis, IV, XIII, 3. Roma-Bari: Laterza, 2008.

${ }^{11}$ Derrida, J. Le siecle et le perdon. In: Le monde des débats, dicembre 1999.

${ }^{12}$ Derrida, ibidem.

${ }^{13}$ Derrida, J. ibidem.

${ }^{14}$ Sêneca, L. A. op. cit., III, XII, 1.
} 
fate del bene e prestate senza sperarne nulla" (Lc 6, 32-35). Questo comandamento impossibile, cui è associata l'idea di un prestito senza speranza di ritorno, sembra essere il solo all'altezza delle spirito di perdono perché, da un lato, radicalizza la critica alla necessità di ricambiare insita nel dono e, dall'altro, sposta l'accento dalla reciprocità alla spontaneità di un gesto unilaterale, che trae la sua sola giustificazione in sé stesso.

La sfida lanciata dalle parole di Gesù è stata accolta tra gli altri da Vladimir Jankélévitch, che nelle sue opere propone "un'etica iperbolica per la quale il perdono è il comandamento supremo; e d'altra parte il male appare sempre al di là. Il perdono è più forte del male e il male è più forte del perdono"15perché "esiste fra l'assoluto della legge dell'amore e l'assoluto della libertà malvagia una lacerazione che non può essere totale"16. Purtroppo difficilmente la storia riuscirà ad affrancarsi dal confronto tra la misura del male radicale e l’immensità del perdono, da questa oscillazione infinita: si arriverà, perfino, a costruire, soprattutto con riferimento alla tragedia della Seconda Guerra Mondiale, la categoria dell'imperdonabile. Sarà proprio lo stesso Jankélévitch ${ }^{17}$ a dare il contributo più significativo dal punto di vista filosofico alla definizione di perdono impossibile, intrecciandola però con la categoria dell'imprescrittibile. Già nel 1956 egli affermava che riguardo all'Olocausto "Tutti i criteri giuridici abitualmente applicabili ai crimini di

\footnotetext{
${ }^{15}$ Intervista a V. Jankélévitch, citata da Gouhier, A., in un articolo intitolato "Le temps de l'imperdonnable et le temps du pardon selon Jankélévitch", pubblicato in. Le Point théologique. Le pardon, atti di un importante convegno dedicato al perdono, organizzato dal Centre Histoire des Idées, Université de Picardie, a cura di M. Perrin. Paris: Beauchesnes, 1987.

${ }^{16}$ Jankélévitch, V. Perdonare? In: L’imprescrittibile. Firenze: La Giuntina, 1987. p. 10.

${ }^{17}$ É interessante seguire l'evoluzione del pensiero di V. Jankélévitch: in un primo saggio pubblicato nel 1956 sotto il titolo di L'imprescriptible, nell' ambito delle polemiche relative alla prescrizione dei crimini hitleriani, si schierò contro ogni possibilità di perdono; nello studio del 1967 Le pardon (tr.it.Il perdono, I. P. L., Milano, 1968), riflettendo a livello filosofico sul tema, costruì la sua etica iperbolica a favore dell'atto di perdonare e identificò il tempo del perdono con il tempo dell'oblio; seguì una terza opera Pardonner? in cui mise in evidenza la necessità della richiesta di perdono affinché quest'ultimo possa essere concesso.
} 
diritto comune in materia di prescrizione sono in questo caso insostenibili"18. la prescrizione ${ }^{19}$, in realtà, opera di diritto per tutti i delitti e i crimini senza eccezione e il termine di prescrizione varia a seconda della natura di essi. Tale fenomeno determina l'estinzione dell'azione penale : esso, da una parte, interdice al postulante, passato un certo periodo, di investire il tribunale competente, dall'altra, una volta proposta l'azione giudiziaria ne impedisce qualsiasi proseguimento. A differenza dell'amnistia, che tende a cancellare le tracce psichiche o sociali, come se niente fosse accaduto, la prescrizione evita di considerare le conseguenze penali dell'azione commessa: cioè vengono meno il diritto e anche l'obbligo di perseguire penalmente il colpevole. Se la prescrizione può essere considerata un effetto del tempo, essa si collega in qualche modo all'irreversibilità: costituisce il rifiuto, dopo un certo lasso di tempo arbitrariamente definito, di ripercorrere il tempo all'indietro fino all'atto colpevole. Le tracce di esso non sono cancellate ma è vietato il cammino per giungere fino ad esse. Su questo sfondo va collocata la legislazione che sancisce l’imprescrittibilità ${ }^{20}$ dei crimini contro l'umanità ${ }^{21} \mathrm{e}$, tra

${ }^{18}$ Jankélévitch, V. L'imprescrittibile. Firenze: La Giuntina, 1987. p. 15.

${ }^{19} \mathrm{Nel}$ nostro ordinamento giuridico il decorso di un determinato periodo di tempo (stabilito dalla legge) senza che intervenga la pronuncia di una sentenza di condanna irrevocabile, determina l'estinzione del reato per prescrizione.

La misura costituisce la rinuncia dello Stato all'applicazione della sanzione punitiva sul presupposto del passare del tempo. I reati per i quali è prevista la pena dell'ergastolo sono imprescrittibili. Sulla base della pena prevista per ciascun tipo di reato, l'art. 157 c.p., cosi come riscritto dalla legge n. 251 del 5 dicembre 2005 (c.d. legge ex Cirielli), disciplina il tempo necessario per la prescrizione del reato.

${ }^{20}$ Vedi ad esempio la Convenzione delle Nazioni Unite sull'imprescrittibilità dei crimini di guerra contro l'umanità, adottata il 26 novembre 1968, e la Convenzione europea sull'imprescrittibilità dei crimini contro l'umanità e dei crimini di guerra, adottata il 25 gennaio 1974, che affermano sul piano internazionale il principio di imprescrittibilità.

${ }^{21}$ La prima codificazione dei crimini contro l'umanità nel diritto internazionale positivo è contenuta nello Statuto del Tribunale militare internazionale di Norimberga, il cui articolo 6 li definisce come "lomicidio volontario, lo sterminio, la riduzione in schiavitù, la deportazione e ogni altro atto disumano commesso ai danni di una qualsiasi popolazione civile, prima o durante la guerra, oppure persecuzioni per motivi politici, razziali o religiosi sempre che tali 
di essi, dei crimini di genocidio ${ }^{22}$. Ciò significa che il principio di prescrizione, generalmente applicabile, non può essere invocato: in altre parole si autorizza l'autorità pubblica a perseguire indefinitamente gli autori di siffatti crimini. Fondamentalmente è la gravità estrema di tali atti a giustificare il perseguimento dei colpevoli senza limiti di tempo: al fallace argomento del venir meno della pretesa punitiva a causa dell'effetto meccanico del tempo, si replica che la riprovazione per i crimini considerati non possa cessare mai. Ciò detto, qual è allora il rapporto tra l'imprescrittibile e l'imperdonabile? Sarebbe davvero un errore confondere le due nozioni in quanto mentre una attiene al piano della punizione, l'altra si colloca su quello della colpa: tuttavia molto spesso sono state confuse perché l'enormità dei crimini contro l'umanità e, in particolare del genocidio, infrange il principio di proporzione che regola i rapporti fra la scala dei delitti o dei crimini e quella delle pene e rende qualsiasi pena inappropriata davanti a un crimine così fuori misura, solo in questo senso i crimini in questione costituiscono un imperdonabile di fatto, come sostiene Paul Ricoeur ${ }^{23}$.

Il breve saggio di Vladimir Jankélévitch "Pardonner?” fu scritto proprio sull'onda del dibattito francese del 1964 sull'imprescrittibilità dei crimini hitleriani e dei crimini contro l'umanità : il testo, che vide la luce

atti o persecuzioni - abbiano costituito o meno una violazione del diritto interno del paese in cui sono stati commessi - siano stati perpetrati nell'esecuzione di uno dei crimini rientranti nella competenza del Tribunale, o in connessione con uno di siffatti crimini”.

${ }^{22}$ Il termine genocidio appare per la prima volta nel 1943, nella prefazione della voluminosa opera del giurista Raphael Lemkin, Axis Rule in Occupied Europe: "Con genocidio s'intende la distruzione di una nazione o di un gruppo etnico. Questa nuova parola, coniata per denotare un'antica pratica nel suo sviluppo moderno, è creata dalla parola gènos del greco antico (razza, tribù) e dal latino caedere (uccidere)".

${ }^{23}$ Ricoeur, P. La memoria, la storia, l'oblio. Milano: Raffaello Cortina editore, Milano, 2003. p. 670. 
nel 1971 e che è contenuto ne "L'imprescriptible", conclude in modo deciso per l'impossibilità e per l'inopportunità, addirittura per l'immoralità del perdono, rammentandoci che "Il perdono è morto nei campi della morte"24.

L'autore propugna, innanzitutto, in un appassionato slancio polemico la necessità di non dimenticare ciò che è accaduto: perdonare genererebbe oblio, mentre vi è un dovere del ricordo ${ }^{25}$ nei confronti delle vittime ("Dimenticare questo crimine gigantesco contro l'umanità sarebbe un nuovo crimine contro il genere umano"26). Il suo netto rifiuto è giustificato da due ordini di ragioni: in primo luogo la constatazione che il perdono può essere accordato o che almeno si può considerare la possibilità di accordarlo, solo se esso è domandato, esplicitamente o implicitamente, da parte del colpevole che si pente e confessa la propria colpa ("Il perdono! Ma essi ci hanno mai domandato perdono? Soltanto la disperazione e la solitudine del colpevole darebbero un senso e una ragion d'essere al perdono"27); in secondo luogo l'affermazione che quando il crimine è troppo grave e supera la linea del male radicale non possa più aspirare ad essere perdonato, perché il perdono deve restare dentro i confini dell'umano, configurandosi come un atto di uomini nei confronti di altri uomini ("Per essere esatti, il grande massacro non è un crimine su scala umana; non più delle grandezze astronomiche e degli anni luce"28).

La forza di tali argomentazioni poggia sull'eccezionalità della Shoah, evento senza precedenti né equivalenti, in grado di rappresentare col suo delirante progetto di sterminio degli ebrei da parte dei nazisti la fine di una storia del perdono (o alla maniera di Hegel della storia come

\footnotetext{
${ }^{24}$ Jankélévitch, V. op. cit., p. 40.

${ }^{25}$ Tale dovere è stato posto al centro della letteratura concentrazionaria: vedi ad esempio Levi, P. Se questo è un uomo. Torino: Einaudi, 1981.

${ }^{26}$ Jankélévitch, V. op. cit., p. 19.

${ }^{27}$ Jankélévitch, V. op. cit., p. 40.

${ }^{28}$ Jankélévitch, V. op. cit., p. 22.
} 
perdono) e di una possibilità storica di perdono. Del resto la singolarità della "soluzione finale" raggiunge il limite dell'inespiabile, per il quale non vi è perdono possibile: in questo caso "non si può punire il criminale con una punizione proporzionata al suo crimine: perché rispetto all'infinito tutte le grandezze finite tendono a uguagliarsi; a tal punto che la punizione diventa quasi indifferente; ciò che è accaduto è alla lettera inespiabile"29. Infatti "le reazioni che provoca sono innanzitutto la disperazione e un senso di impotenza davanti all'irreparabile”30.

In altre parole laddove non può esserci espiazione, vi è l'imperdonabile, come se il perdono dovesse acquistare un senso solo attraverso un'assoluzione ottenuta tramite la punizione stessa, su uno sfondo di salvezza, di redenzione, di sacrificio. In realtà dovremmo domandarci se il perdono non debba liberarsi finalmente dalla correlazione tra la possibilità di punire e quella di perdonare ereditata dalla tradizione della semantica religiosa del perdono, che lo collega al pentimento e alla confessione, alla capacità di espiare e di riscattarsi. Dunque è necessario chiedersi se il perdono cominci proprio dove esso sembra finire: si deve e si può perdonare solo l'imperdonabile, l'inespiabile perché tale atto solamente nell'impossibile del possibile trae la sua origine. Spingersi nel cuore di questo paradosso è un'operazione tanto complessa quanto affascinante.

Derrida, riprendendo il pensiero di Jankélévitch nella sua opera dal titolo "Perdonare", propone non solo di distinguere il più finemente e rigorosamente possibile tra l'imperdonabile (come concetto filosofico), da una parte e l'imprescrittibile (come concetto giuridico), dall'altra, ma anche di far luce sulle nozioni affini e tuttavia differenti di irreparabile, incancellabile, irrimediabile, irreversibile, indimenticabile, irrevocabile, inespiabile. Egli sottolinea che malgrado le decisive differenze che le

\footnotetext{
${ }^{29}$ Jankélévitch, V. ibidem.

${ }^{30}$ Jankélévitch, V. ibidem.
} 
separano, tutti questi concetti hanno in comune una negatività, il "non" di un impossibile che significa alternativamente o contemporaneamente “impossibile perché non si può”, “impossibile perché non si deve”. In tutte le situazioni descritte non si deve e/o non si può ritornare su di un passato che non passa ${ }^{31}$, perché l'evento ha avuto luogo e qualcuno, ormai, si è macchiato della colpa: la memoria di questo passato resta irriducibile, intrattabile in quanto esso non si lascia mai ridurre, modificare, modalizzare in un presente passato o in un passato presentabile o ripresentabile. Una delle differenze principali con il dono, che si accorda più facilmente al tempo presente, è proprio questa. Senza questo privilegio ostinato del passato che rimane e permane nella costituzione della temporalizzazione, non vi è possibilità di perdonare, a meno che il desiderio e la promessa del perdono, se non addirittura della riconciliazione e della redenzione, non significhino segretamente questa rivolta o questa rivoluzione contro una temporalizzazione, addirittura una storicizzazione che ha senso solo se si considera l'essere stato del passato. L'esperienza del perdono diventa così una struttura essenziale e ontologica (non soltanto etica o religiosa) del rapporto soggettivo e intersoggettivo con il tempo: rappresenta l'essere del tempo poiché comporta un passato irrecusabile e immodificabile.

Tuttavia l'essere passato di qualcosa che è accaduto non basta ancora per costruire un concetto del perdono da domandare o da accordare: non è sufficiente che ci sia stato semplicemente un fatto (participio passato che indica un fare, un'azione che ha avuto luogo e resta irrecusabile), ma occorre che tale fatto sia un misfatto, un male, un torto compiuto da qualcuno (il responsabile) nei confronti di qualcun'altro (la vittima).

${ }^{31} \mathrm{Si}$ richiama così l'espressione usata da Rusconi, G. E. In Germania: un passato che non passa. I crimini nazisti e l’identità tedesca. Torino: Einaudi, 1987. 
Jacques Derrida ci ricorda che "tra dono e perdono esiste almeno quest'affinità o quest'alleanza, oltre la loro incondizionalità di principio,l'uno e l'altro hanno un rapporto essenziale con il tempo, con il movimento della temporalizzazione. E tuttavia legato a un passato che in un certo senso non passa il perdono resta un'esperienza irriducibile a quella del dono, di un dono che si accorda più facilmente al presente, alla presentazione o alla presenza del presente"32. Dunque dono e perdono hanno secondo il celebre filosofo francese due soli caratteri in comune: una connotazione temporale (seppure diversa) e, infine, l'incapacità di essere sottoposti a condizioni che ne determinino l'esistenza. Almeno per il perdono è stata prospettata, però, la necessità di una richiesta e di una correlazione tra quest'ultima e la concessione del perdono stesso: in buona sostanza si può perdonare solo chi ha domandato prima perdono o si deve perdonare anche chi non riconosce la sua colpa? Il legame tra perdono chiesto e perdono accordato, sebbene suffragato dalla tradizione religiosa ${ }^{33}$ in materia, non sembra essere un requisito fondamentale dell'atto in questione, che rientra nella discrezionalità di una scelta volontaria, senza secondi fini.

Una volta affermato il carattere non condizionale del perdono occorre però chiedersi: a chi spetta il potere di perdonare? E chi deve essere perdonato? Uscire dal circuito vittima-carnefice sembra più difficile di quanto si pensi, soprattutto se si considera il fenomeno recente delle scuse da parte di Capi di Stato nei confronti di intere collettività, che hanno subito i più diversi abusi. Lo studioso Maurizio Bettini ha definito simili richieste con l'espressione "perdono storico"34, sottolineando così il fatto che esse si riferiscono quasi sempre a episodi non del passato recente,

\footnotetext{
${ }^{32}$ Derrida, J. Perdonare. Milano: Raffaello Cortina Editore, 2004. p. 22-23.

${ }^{33}$ Catechismo della Chiesa Cattolica: "Gli atti del penitente richiesti per ottenere l'assoluzione da parte del sacerdote sono: il pentimento, la confessione o manifestazione dei peccati al sacerdote e il proposito di compiere la soddisfazione e le opere di soddisfazione".

${ }^{34}$ Bettini, M. Sul perdono storico. Dono, identità, memoria e oblio. In: Flores, M. (a cura di). Storia, verità, giustizia. Milano: Mondadori, 2001. p. 20-21.
} 
ma del passato lontano ${ }^{35}$ e che appaiono, quindi, svincolate dalle necessità dettate dal presente, come la legittima esigenza di giustizia delle vittime o il giudizio dei contemporanei che hanno assistito ai fatti in questione. Le scene pubbliche di penitenza e di contrizione ${ }^{36}$, appena richiamate, suscitano, al di là del sospetto di banalizzazione e di teatralizzazione, una questione di legittimità: di quale delega possono avvalersi un uomo politico nelle sue funzioni, il capo attuale di una comunità religiosa per domandare perdono a vittime delle quali, del resto, non sono i personali aggressori e le quali eventualmente non hanno personalmente sofferto del torto considerato? Si pone una questione di rappresentatività nel tempo e nello spazio lungo la linea di continuità di una tradizione ininterrotta. Tanto è vero che Klaus M. Kodalle proprio all'inizio delle sue riflessioni sulla dimensione pubblica del perdono si domanda: " $i$ popoli sono capaci di perdonare?37", interrogativo che ne richiama subito un altro: i popoli possono chiedere perdono?. La risposta è senz'altro negativa, sia perché il vero perdono è un atto essenzialmente individuale che non ammette rappresentanza, compiuto dalla vittima ${ }^{38}$ nei confronti del suo offensore, sia perché la facoltà di perdonare in quanto esercizio di memoria collettiva, come evidenziato in precedenza nella nostra trattazione, può purtroppo essere piegata alle necessità storiche e politiche di un dato momento presente, a scapito della sua sincerità. La collettività non ha coscienza morale: gli atti possono essere imputati solo agli individui singolarmente considerati e solo ad essi può essere rimessa la colpa.

\footnotetext{
${ }^{35}$ Bettini, M. Ibidem.

${ }^{36}$ Una discussione storica accompagnata dall'analisi di casi di richieste di perdono in Brooks, R. L. The age of apology. In: Brooks, R. L. (a cura di), When Sorry Isn't Enough. New York: University Press, 1999. p. 3-11.

${ }^{37}$ Kodalle, K. M. Verzeihung nach Wendezeiten? (conferenze inaugurali tenute all'Università F. Schiller di Iena, 2 giugno 1994), Palm ed Enke, Erlangen e Iena, 1994, p. 36.

38 "Spetta alle vittime perdonare” sostiene Jankélévitch, V. Op.cit., p. 44.
} 
I popoli ricadono continuamente nella rimuginazione dei vecchi rancori, delle antiche umiliazioni: in alcuni casi il male fatto ha generato una spirale inesauribile di vendette o ritorsioni, in altri esso resta un'inguaribile e non accettata ferita in chi non ha saputo impedirlo. Kodalle, di fronte alla riproposizione dell'odio anche su scala collettiva, propone alle memorie malate come rimedio l'idea di una normalità nei rapporti tra vicini nemici, che non solo costituirebbe l'incognito del perdono (Inkognito der Verzeihung), ma sarebbe non il risultato di un processo di fraternizzazione, bensì di una correzione nello scambio di relazioni, basata sull'applicazione della cultura della considerazione (Nachsichtlichkeit) in ambito civico e cosmopolitico. Essa può essere messa a confronto sul piano della responsabilità morale con gli odi ereditari nella forma di una tenace volontà di comprendere quegli altri, che la storia ha reso dei nemici; implica, inoltre, se riferita a sé stessi il rifiuto della discolpa a buon mercato rispetto allo straniero nemico o ex nemico. A questo livello la benevolenza passa, in particolare, attraverso un'attenzione agli eventi fondatori dell'identita altrui e ai racconti di vita dell'altra parte: è necessario "imparare a raccontare altrimenti”, mettersi nei panni dell'altro, guardare alle vicende storiche anche dal suo punto di vista.

Non si può certo dimenticare, però, che il presupposto esistenziale del perdono è l'esperienza della colpa, posta da Jean Nabert accanto a quella dello scacco e della solitudine fra "i dati della riflessione"39 (ossia fra "i sentimenti che alimentano la riflessione, ne costituiscono la materia" ${ }^{\prime \prime}$ ) oppure situata da Karl Jaspers tra "le situazioni limite" ", vale a dire quelle determinazioni non fortuite dell'esistenza che noi troviamo sempre già là, quali la morte, la sofferenza, la guerra. Sul piano fattuale, la colpa consiste

\footnotetext{
${ }^{39}$ Nabert, J. Elementi per un'etica. Padova: La Garangola, 1975; "I dati della riflessione” è il titolo del I capitolo.

${ }^{40}$ Nabert, J. Op. cit., p. 8.

${ }^{41}$ Jaspers, K. Chiarificazione dell'esistenza. Milano: Mursia, 1978.
} 
nella trasgressione di una regola, quale che essa sia, di un dovere, che comporta delle conseguenze evidenti, e cioè fondamentalmente un torto fatto a un altro. Si tratta di una cattiva azione e a questo titolo condannabile in termini di apprezzamento negativo. Nel vocabolario del saggio kantiano sulle grandezze negative, la colpa è una grandezza negativa della pratica ${ }^{42}$ : essa sembrerebbe quindi tanto limitata quanto la regola che infrange, anche i suoi effetti, per le loro ripercussioni in termini di sofferenza inflitta, possono rivestire un aspetto indefinito. In realtà l'implicazione dell'agente nell'atto equivale a "rendere illimitata la risonanza sulla coscienza di ognuna delle nostre azioni" ${ }^{\prime 3}$. Se è vero, quindi, che la colpa appartiene inevitabilmente alla nostra condizione storica occorre designare la struttura fondamentale nella quale essa viene a inscriversi: l’imputabilità delle nostre azioni. In effetti non può esserci perdono se non là dove si può accusare qualcuno, presumerlo o dichiararlo colpevole di un'offesa, ritenendolo così il suo vero autore. Ricoeur considera l'imputabilità addirittura una dimensione fondamentale "dell'uomo capace "4": questo collegamento fra l'atto e l'agente, fra il "che cosa” dell'azione e il "chi” della potenza d'agire può essere in qualche modo toccato, ferito da una penosa affezione, il senso di colpa.

La forma specifica, che l'attribuzione della colpa a sé stessi assume, è la confessione, ovvero l'atto di linguaggio attraverso il quale un soggetto prende su di sé, assume l'accusa. Confessare qualcosa implica necessariamente la capacita di ricordare nella misura in cui la rimemorazione si attesta come una potenza di legame creatore di storia. La memoria è, in via di principio, innocente, mentre la confessione supera il dubbio tra l'innocenza e la colpa, oltre a legare l'atto al suo agente. Ciò che è in gioco è "dietro la qualità della sua azione, la qualità della causalità dalla quale è proceduta nella sua

\footnotetext{
${ }^{42}$ Kant, I. Tentativo per introdurre nella filosofia il concetto delle quantità negative, in Scritti precritici, a cura di Carabellese, P.; Assunto, R.; Hohenemser, R. Bari: Laterza, 1953. p. 257-301.

${ }^{43}$ Nabert, J. Op. cit., p. 11.

${ }^{44}$ Ricoeur, P. op. cit., p.653.
} 
azione" ${ }^{45}$. A questo livello di profondità il riconoscimento di sé è indivisibilmente azione e passione, azione di agire male e passione di essere affetti dalla propria azione: in virtù di ciò non si può più "dissociare l'idea della propria causalità dal ricordo dell'atto singolare che essa ha compiuto"46.

Il coraggio di domandare perdono sembrerebbe derivare dalla nostra capacità a dominare il corso del tempo secondo Hannah Arendt, che nella sua Vita activa individua tre attività fondamentali nella vita dell'uomo: lavoro, opera e azione. Quest'ultima si distingue dagli altri due termini in virtù della sua temporalità propria: mentre il lavoro si esaurisce nella consumazione e l'opera vuole durare di più dei suoi autori mortali, l'azione vuole semplicemente continuare, si dispiega in uno spazio di visibilità pubblica caratterizzato da una fitta rete di relazioni e interazioni, nonché dalla dimensione originaria della pluralità umana. Il potere di perdonare e di promettere fanno fronte alle debolezze insite nella pluralità. La fragilità degli affari umani, in effetti, non si riduce al carattere perituro, mortale, di imprese sottoposte all'impietoso ordine delle cose, alla cancellazione fisica delle tracce data dall'oblio, ma attiene anche al carattere di incertezza, connesso al fenomeno dell'azione sotto la condizione della pluralità. Tale incertezza deve essere rapportata, da una parte, all'irreversibilità che rovina il voto di dominio sovrano applicato alle conseguenze dell'azione, a cui replica il perdono; dall'altra, all’impredicibilità che compromette la fiducia nell'aspettativa di un corso di azioni, l'affidabilità dell'agire umano, a cui si contrappone la promessa ${ }^{47}$. "Il rimedio contro l'irreversibilità $e$

\footnotetext{
${ }^{45}$ Nabert, J. ibidem.

${ }^{46}$ Nabert, J. ibidem.

${ }^{47}$ F. W. Nietzsche allinizio della seconda dissertazione della Genealogia della morale si chiede: "Allevare un animale, cui sia consentito far delle promesse, non è forse precisamente questo il compito paradossale impostosi dalla natura per quanto riguarda l'uomo?": l'autore sottolinea come la memoria, che conferisce all'uomo il potere di mantenere le proprie promesse, lo renda "calcolabile, regolare necessario" e così "capace di rispondere di sé come avvenire"in Genealogia della morale. Milano: Bur, 1997. p. 255.
} 
l'imprevedibilità del processo avviato dall'azione non scaturisce, da un'altra facoltà superiore, ma è una delle potenzialità dell'azione stessa"48 ribadisce l'autrice nella sua opera. Il perdono ("facoltà di perdonare") e la promessa ("facoltà di fare e mantenere delle promesse") costituiscono facoltà umane che dipendono dalla pluralità: si fondano su esperienze che nessuno può fare nella solitudine e che richiedono necessariamente la presenza dell'altro, la loro sfera di esercizio è del resto eminentemente politica. Infatti "le due attività si completano poiché una, il perdonare, serve a distruggere i gesti del passato, $i$ cui "peccati" pendono come la spada di Damocle sul capo di ogni nuova generazione; e l'altra, il vincolarsi con delle promesse, serve a gettare nell'oceano dell'incertezza, quale è il futuro per definizione, isole di sicurezza senza le quali nemmeno la continuità, per non parlare di una durata di qualsiasi genere, sarebbe possibile nelle relazioni tra gli uomini"49. Anzi Hannah Arendt reputa che proprio Gesù nei Vangeli ha sottolineato il carattere umano, prima ancora che divino, del perdono, quando in primo luogo ha affermato contro scribi e farisei che non solo Dio ha il potere di perdonare e, in secondo luogo, ha ribadito che tale potere non deriva da Dio (come se Dio soltanto perdonasse, attraverso la mediazione degli esseri umani), ma al contrario va praticato dagli uomini gli uni verso gli altri, affinché essi possano sperare di essere perdonati anche da $\mathrm{Dio}^{50}$. Anche se l'autrice reinserisce così il perdono all'interno del circuito della reciprocità: unicamente chi è capace di perdonare l'altro, potrà fare affidamento sulla possibilità di essere perdonato tanto da Dio quanto dai fratelli.

\footnotetext{
${ }^{48}$ Arendt, H. Vita activa. La condizione umana. Milano: Bompiani, 2008. p. 175.

${ }^{49}$ Arendt, H. Ibidem.

${ }^{50}$ Si legge, infatti, nei Vangeli: "Così anche il mio Padre celeste farà a ciascuno di voi, se non perdonerete di cuore al vostro fratello"(Mt 18,35); "Se voi, infatti, perdonerete agli uomini le loro colpe, il Padre vostro celeste perdonerà anche a voi; ma se voi non perdonerete agli uomini, neppure il Padre vostro perdonerà le vostre colpe"(Mt 6, 14-15); "Se tuo fratello pecca, rimproveralo; ma se si pente, perdonagli. E se pecca sette volte al giorno contro di te e sette volte ti dice: Mi pento, tu gli perdonerai” (Lc 17,3-4).
} 
Infatti, "solo attraverso questa costante mutua liberazione da ciò che fanno gli uomini possono rimanere agenti liberi" ${ }^{\prime}$. La conferma di ciò è data, da una parte, dall'opposizione fra perdono e vendetta, queste due maniere umane di reagire all'offesa; dall'altra parte dal parallelismo fra perdono e punizione, laddove entrambe interrompono la sequela senza fine dei torti. Per questo motivo "il perdono è l'esatto opposto della vendetta, che consiste nel reagire a un'offesa originale, e lungi dal porre un termine alle conseguenze del primo errore, lega ognuno al processo, permettendo alla reazione a catena implicita in ogni azione di imboccare un corso sfrenato. Diversamente dalla vendetta, che è la naturale, automatica reazione alla trasgressione e che per effetto dell'irreversibilità del processo dell'agire può essere prevista e anche calcolata, l'atto del perdonare non può mai essere previsto; è la sola reazione che agisca in maniera inaspettata e che quindi ha in sé, pur essendo una reazione, qualcosa del carattere originale dell'azione. Perdonare in altre parole è la sola reazione che non si limita a re-agire, ma agisce in maniera nuova e inaspettata. La libertà contenuta nell'insegnamento di Gesù è la libertà dalla vendetta che imprigiona chi fa e chi soffre dell'automatismo implacabile del processo dell'azione, che non ha in sé alcuna tendenza a finire. L'alternativa al perdono, ma non il suo opposto, è la pena, che ha in comune col primo il tentativo di porre un termine a qualcosa che senza interferenza potrebbe proseguire indefinitamente. $\grave{E}$ quindi significativo (un elemento strutturale nella sfera delle faccende umane) che gli uomini siano incapaci di perdonare ciò che non possono punire e di punire ciò che si è rivelato imperdonabile"52.

In definitiva, tutto si gioca sulla possibilità di separare l'agente dalla sua azione sebbene Nicolai Hartmann sostenga che "L'essere colpevole di un'azione cattiva non può essere tolto a nessuno, perché è indivisibile dal

\footnotetext{
${ }^{51}$ Arendt, H. Op.cit., p. 177.

${ }^{52}$ Arendt, H. Op. cit., p. 177-178.
} 
colpevole ${ }^{3}$. Certamente possiamo attenuare il rimorso, ma non la colpevolezza stessa: "Vi è certo moralmente un superamento del male...ma non una nullificazione della colpa come tale"54. In altre parole "la colpa è per essenza imperdonabile non soltanto di fatto, ma di diritto"55 come sostiene Paul Ricoeur. Il legame tra la colpa e il sé, tra la colpa e l’ipseità sembra indissolubile. La conseguenza principale dovrebbe essere il fatto che il colpevole sia reso in tal modo capace di ricominciare perché nell'originaria disposizione dell'uomo al bene, riconosciuta da Immanuel Kant, risiede la possibilità del suo "ristabilimento operato con le nostre proprie forze" ${ }^{\text {": }}$ : sotto il segno del perdono il colpevole può essere ritenuto capace di qualcosa d'altro che dei suoi delitti, può ricominciare, senza cancellare nulla, ma facendosi carico dei suoi sbagli. Secondo Eligio Resta compiono, infatti, un errore grossolano le istituzioni "quando ritengono che un individuo è per sempre quello che ha fatto in un momento annullando ogni tragitto intertemporale e la molteplicità degli "io" che egli è stato" 57 . La vera sfida consiste, quindi, nel perdonare l'offensore pur condannando la sua azione, come ribadisce Jacques Derrida.

Eppure il perdono ha anche un altro merito: quando è autentico riesce a interrompere la catena del risentimento e a liberare la vittima, altrimenti imprigionata per sempre nel suo ruolo paranoico. D’altronde è ancora Vladimir Jankélévitch a sostenere che laddove non si può perdonare "si può almeno risentire inesauribilmente" 58 , provare "un sentimento rinnovato e intensamente vissuto della cosa inespiabile" ${ }^{\text {, }}$, che non si chiama

\footnotetext{
${ }^{53}$ Hartmann, N. Etica III: metafisica dei costumi. Napoli: Guida Editore, 2009. p. 248.

${ }^{54}$ Hartmann, N. Op.cit, p.249.

${ }^{55}$ Ricoeur, P. Op. cit., p. 670.

${ }^{56}$ Kant, I. La religione entro i limiti della sola ragione. Roma-BariL Laterza, 1980. p. 54.

${ }^{57}$ Resta, E. Il diritto fraterno. Roma-Bari: Laterza, 2002. p. 119.

${ }^{58}$ Jankélévitch, V. Op. cit., p. 49.

${ }^{59}$ Jankélévitch, V. Ibidem.
} 
rancore, ma "orrore insormontabile di ciò che è accaduto" 60 . L'orrore spinge a distogliere lo sguardo da qualcosa la cui vista è umanamente insopportabile, proprio come le barbarie del Novecento.

Paul Ricoeur continua a metterci in guardia: "Il perdono se ha un senso e esiste, costituisce l'orizzonte comune della memoria, della storia e dell'oblio. Sempre in ritirata, l'orizzonte sfugge alla presa. Esso rende il perdono difficile: né facile, né impossibile" è quello che, prendendo sul serio il tragico dell'azione, punta alla radice degli atti, alla fonte dei conflitti e dei torti che richiedono il perdono: non si tratta di cancellare un debito sulla tabella dei conti, a livello di un bilancio contabile, si tratta di sciogliere dei nodi" ${ }^{2}$, che, ribadiamo, sono tanti e spesso inestricabili. È necessario tagliare il legame tra il colpevole e il male compiuto, ma anche tra l'offeso e il male sofferto. In effetti, come evidenziato in precedenza, perdonare vuol dire far si che il tempo riprenda a scorrere tanto per la vittima quanto per il carnefice, inchiodati altrimenti al momento dell'offesa, chiusi nella fissità immobile di un torto passato, sul quale non si può più agire perché appartenente al già stato.

In altri termini, l'atto di porre "il reale al passato" ${ }^{3}$, per riprendere un'espressione di Sartre, comporta innanzitutto una metanoia ${ }^{64}$, una trasformazione dall'interno, un cambiamento interiore, mentale appunto, frutto tanto del "lavoro di rimemorazione" ${ }^{65}$ (Erinnerungsarbeit), quanto

\footnotetext{
${ }^{60}$ Jankélévitch, V. Ibidem.

${ }^{61}$ Ricoeur, P. Op.cit., p. 675.

${ }^{62}$ Ricoeur, P. Ibidem.

${ }^{63}$ Sartre, J. P. Immagine e coscienza. Psicologia fenomenologica dellimmaginazione. Torino: Einaudi, 1964.

${ }^{64}$ Resta, E. Op.cit., p. 123.

${ }^{65}$ Freud, S. Ricordare, ripetere e rielaborare. In Opere, vol. VII. Torino: Bollati Boringhieri, 1977.
} 
del "lavoro del lutto"66, propugnati entrambi dalla psicanalisi. Da una parte, occorre ricordare perché l'oblio rappresenterebbe un inutile tentativo di rimozione: le tracce cancellate, infatti, hanno l'inesauribile capacità di ripresentarsi continuamente sotto altre forme e di dar vita a patologie mascherate; dall'altra, è necessario elaborare il dolore per la perdita della possibilità di modificare il passato: accettare una volta per tutte ciò che è accaduto sembra l'unica strada percorribile se si vuole superare il male e non restare incatenati indefinitamente ad esso.

Hegel nella Fenomenologia dello spirito ritiene il perdono il motore della storia e lo nomina, per la prima volta, quando parla di "riconciliazione", intesa come "un riconoscere reciproco che è lo spirito assoluto" ${ }^{\circ}$ : egli afferma che "il Sì della conciliazione in cui $i$ due Io dimettono il loro opposto esserci, è l'esserci dell'Io esteso fino alla dualità, Io che quivi resta uguale a sé e che nella sua completa alienazione e nel suo completo contrario ha la certezza di sé stesso"68. Eppure perdonare vuol dire spingersi al di là di ogni possibilità di riconciliazione, la sua vera essenza non risiede nello consentire alla vittima di riconoscere il carnefice e viceversa, ma nel superare ogni possibile limite, in quell'andare sempre oltre le norme, i criteri, le regole, che lo rende "iperbolicamente etico"69.

In sintesi, il perdono può essere definito come un atto di memoria in grado di interrompere e, nello stesso momento, di far ripartire ogni volta in modo diverso la corrente ordinata della temporalità storica: occorre che il richiamo al passato sia presente nella ferita con la stessa forza che aveva nell'istante in cui essa è stata inferta. Il perdono, sebbene costituisca " $u$ n

\footnotetext{
${ }^{66}$ Freud, S. Lutto e malinconia. In: Opere, vol. VIII. Torino: Bollati Boringhieri, 1977.

${ }^{67}$ Hegel. Fenomenologia dello spirito. Firenze: La Nuova Italia, 1996. p. 391.

${ }^{68}$ Hegel. Op. cit., p. 415.

${ }^{69}$ Derrida, J. Sulla parola. Roma: Nottetempo, 2004. p. 76.
} 
potere riservato a tutti e posseduto da tutti" ${ }^{\prime \prime 0}$, è e deve rimanere eccezionale e straordinario: non potrà mai essere né normale, né normativo, nonostante i numerosi tentativi del diritto di incorporarlo e, soprattutto, non dovrà nascondere la sua estrema fragilità.

Recebido em: 29/8/2012

Aprovado em: 29/8/2012

Autor convidado e artigo não avaliado

${ }^{70}$ Canetti, E. Massa e potere. Milano: Adelphi, 1981. p. 310. 